\title{
APPLICATION OF ADVANCED ENERGY STORAGE IN RENEWABLE ENERGY POWER GENERATION
}

\author{
Fengwei Li ${ }^{1}$, Gang $\mathrm{zhu}^{2^{*}}$ \\ ${ }^{1}$ Department of Water Conservancy and Architecture, Beijing Agricultural Vocational College, Beijing, 100000, China. \\ ${ }^{2}$ College of Mechanical and Electrical Engineering, Beijing Agricultural Vocational College, Beijing, 100000, China. \\ *Corresponding authors e-mail: zhugang1367118@126.com(GZ)
}

This is an open access article distributed under the Creative Commons Attribution License, which permits unrestricted use, distribution, and reproduction in any medium, provided the original work is properly cited

\section{ARTICLE DETAILS}

\section{Article History:}

Received 12 November 2017 Accepted 12 December 2017 Available online 1 January 2018

\section{ABSTRACT}

This work to the introduction of the concept of generalized momentum power system, through the establishment of the flywheel energy storage system and super capacitor and generalized momentum of renewable energy power generation system model, the generalized momentum compensation control method is proposed. The flywheel energy storage device and super capacitor connected to the renewable energy power generation system of central point voltage, real-time bus voltage to test the voltage of the central point, flywheel angular velocity, computational power system equivalent inertia parameters and the instantaneous momentum flywheel energy storage devices. Calculate the momentum increment of the renewable energy system relative to the standard state; The angular velocity of the flywheel is adjusted so that the momentum increment of the flywheel storage device is equal to the momentum increment of the renewable energy generating system. This method will flywheel energy storage and super capacitor technique is applied to renewable energy power generation system can solve the energy state of renewable energy power generation system, real-time measurement and calculation as well as the energy storage system and energy exchange between the renewable energy generation systems control method.

\section{KEYWORDS}

Energy storage, super capacitor, calculate, renewable.

\section{INTRODUCTION}

The Times tide, such as the history of the general rolling from the wheel, technology soar, with each passing day. Economic development, people's living standards are increasing. But at the same time, also rushed to see this unsightly pollution, think about this irreparable energy and consumption. Electricity, as the most common convenience, the energy used by people to rely mainly on chemical fuel combustion to transformation, despite the long efforts and exploration, is still unable to avoid some of the pollution of gas and greenhouse gas emissions, and nonrenewable energy end The With the development of the times, new energy sources such as solar energy and wind energy are gradually recognized by people, they do not discharge any harmful substances, there is no immigration problem, so the development of wind power is to reduce greenhouse gas emissions, protect the ecological environment, improve the power structure Important measures.

At present, practical energy storage technology has battery energy storage, superconducting energy storage, flywheel energy storage and fuel cell storage, etc., including battery energy storage technology has a long history, widely used, technology is very mature, the battery as the most investment / cost One of the energy storage technology, with a modular, fast response, the high degree of commercialization. Battery storage system installation flexibility, construction cycle is short, there are many successful applications in the power system, but there are some problems [1-5].

Lithium-ion battery single output voltage is high, wide operating temperature range, high specific energy, high efficiency, low self-discharge rate, State Grid Corporation and China Southern Power Grid Corporation also established a megawatt-class lithium-ion battery energy storage power station, to carry out related Demonstration study. But it is overcharge, internal short circuit, etc. will occur under the temperature rise, there is a certain security risk. lithium battery

In the charge and discharge of large random and frequent charging applications, the cycle life is still restricting the application of lithium-ion battery. Sodium sulfur battery is working in the vicinity of the high temperature of $300^{\circ} \mathrm{C}$ battery, high energy, high efficiency, almost no selfdischarge, deep discharge performance, in the power storage application is more mature. However, high-temperature operation of sodium and sulfur batteries, the need for strict temperature control; electrode active material in the molten state, electrolyte preparation and stability of demanding, there are also high security risks. All vanadium flow battery almost no self-discharge, long cycle life, power and capacity can be independently designed, capacity scalability, suitable for large-capacity energy storage applications [6-11].

\section{SELF-GENERATING WIRELESS CHARGING SYSTEM}

In view of the bad weather, we must take into account the various circumstances, such as when the super capacitor in the exhaustion of electricity, we hereby in this taxi to join a number of self-generating devices, which also known as joint power generation.

\subsection{Piezoelectric power generation foot pad}

China is a populous country, that the car is an important means of transport of national travel, so the taxi up and down the frequent flow of people, people up and down the taxi and sit when the taxi must be stepping on the footpad up and down, for which we also specifically in the taxi Front and rear doors on and off the foot pad implanted in the piezoelectric ceramic, through the pressure can also generate electricity, the circuit rectifier and regulator access to the super capacitor to go, to the super capacitor charge. 
Our aim is using the piezoelectric chip strain to obtain the current, the current from the rectifier circuit into DC, and then after DC boost circuit output stable DC voltage, and then power management chip to control the lithium battery charge. However, the piezoelectric device produces only a few microamps, which cannot directly drive the DC boost circuit, so we use rectifier circuit rectifier, and get a pulse DC voltage, that see the voltage of the oil filter circuit output a stable DC voltage, and then super Capacitor charging, the previous part of the role of buffer power. And then drive the DC boost circuit and power management circuit work, by the lithium-ion battery to produce the energy collected. Our ultimate goal is to transform tiny vibrations into viable energy that can be exploited by us.

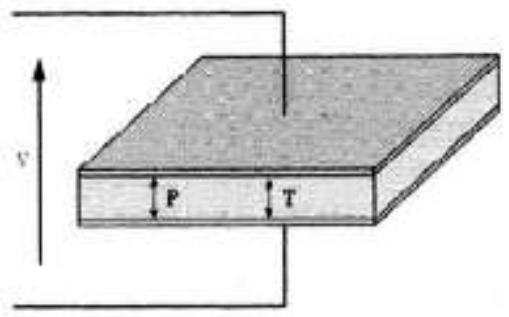

Figure 1: Schematic diagram of the piezoelectric device

In general, there are two modes of operation in the direction of the piezoelectric material, which are the length vibration mode and the transverse vibration mode. We use the transverse vibration mode, the direction $\mathrm{T}$ of the externally applied stress is parallel to the polarization direction $\mathrm{P}$, The resulting voltage is a polarized directional piezoelectric element.

\subsection{Thermoelectric conversion}

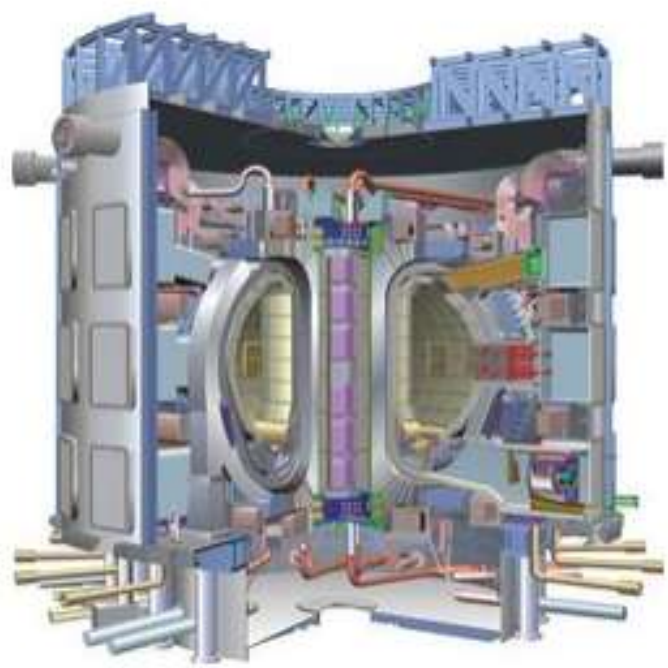

Figure 2: Heat-to-electrical conversion module

The heat-to-electrical conversion module with the highest power generation capability in the world has been successfully developed this year to convert heat energy directly into electrical energy without going to have to undergo mechanical energy conversion (turbine or turbine, generator) as in conventional thermal power plants. Its power generation principle is based on the Seebeck phenomenon, that is, in the same loop, by the different bimetallic two connection points between the temperature difference between the voltage, the heat of an electrical semiconductor components of the upper and lower temperature difference between the positive and negative Potential difference, thus generating electricity. This type of power generation device does not exist in the drive part of the static state of the new power generation mode. There will be no fault. Its temperature performance up to $500{ }^{\circ} \mathrm{C}$, low temperature side is only $20^{\circ} \mathrm{C}$, the maximum output power density up to $1 \mathrm{w} / \mathrm{cm}$, more than just one module can generate $15 \mathrm{w}$, an area of only $14.4 \mathrm{~cm} 2$, for which we will use the Sebeck effect In the car's engine, through the engine's motion heat and the combination of Sebeck effect of the engine heat conversion into electricity will be recycled, and then connected with the super capacitor to achieve the energy storage, easy to use electric vehicles.
In addition to the use of this shell, but also inexhaustible huge solar energy to generate electricity. In addition to the use of light-volt effect of a direct conversion of light to electricity power generation mode, the other solar power generation method that is the use of Seibeck effect of a precise thermal conversion of electricity. So as to achieve the comprehensive utilization of energy.

\subsection{Solar roofs}

We have the top of the car covered with solar panels, which can provide the daily use of electronic equipment within the vehicle, and the use of equipment in the case of surplus can also generate electricity to the super capacitor, so that cars can use the super capacitor of electricity, thus Make it run farther. Solar panels added to the top of the car can be effectively integrated use of space to achieve optimization.

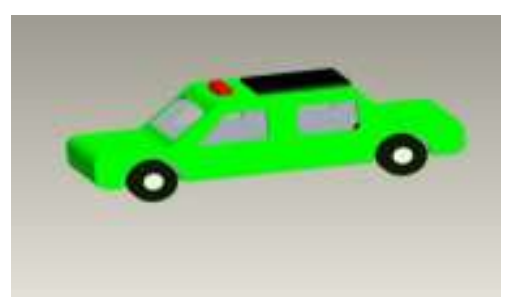

Figure 3: Solar roof

Because of our country's vast geographical regions, China's resources to be too much radiation will be based on regional and weather changes are somewhat different, due to the northern weather conditions change little, we have to the north of solar radiation and combined with different light Angle and the connection between the solar panels as an example: in the fixed light intensity of the output power of the photovoltaic cell depends on the load resistance $\mathrm{R}$, the output power of solar cells in the best load resistance $\mathrm{R}$ reached the maximum power:

$$
\mathrm{P}=\mathrm{U} \times \mathrm{I}
$$

However, in the actual use of solar cells, the direction of solar panels and the angle of solar light on the power generation efficiency of the battery plate has a profound impact, for which we experimental results showed in Figure 3. Experiments show that when the incident light is parallel to the normal of the battery plate (ie, the incident light is irradiated with the solar panel), the voltage generated by the solar panel is the most efficient, and the power is gradually reduced as the irradiation angle increases.

At the same time, the sun at different times in the day the intensity of the sun was experimented, the experiment in the sun and solar panels parallel to the normal, that is, vertical sunlight, to explore the efficiency of solar cells in the day. The exact trend of the law shown in Figure 4. It can be seen from the figure, between 12:00 noon to two o'clock in the afternoon, the solar power generation capacity should be the strongest, with the sun tilt angle increases, the efficiency gradually decreased.

Because of the electrical loss makes the combination of solar panels is different, the possible power and its efficiency are also atypical, so in the experiment, for the different series of parallel arrangement of the battery panels were related experiments. There are five combinations of series and parallel. Measurements are perfect for each type of connection. Respectively, the measurement of discrete angles of exposure, measurement time, each connection state is to take the maximum measurement. The following is the five types of connection plate.

Table 1 for the five connections, the volt-ampere characteristics of the panel, the experiment for each connection are used at different angles of exposure, obtained a unique data. For each connection, in the case of open circuit, measure its output voltage $\mathrm{V}$, and then in the case of short circuit to measure its output current I. According to the volt-ampere characteristics, the power curve under different connection modes is plotted.

Based on the measured data, the solar power generation efficiency of the five connections is calculated and the results are shown in the figure.

It can be seen from the experimental results that in the five different combinations, the combination of four parallel combinations of batteries provides the strongest current, the combination of four series of battery voltage to provide the strongest, four-series parallel and four parallel Power is greatest. In order to optimize the efficiency, so we chose the four strings in parallel as our bus top connection. 
In 2016 the country has about 1 million taxis.

Standard Taxi Size: $4945^{*} \mathrm{~mm} 1845 \mathrm{~mm} * 1480 \mathrm{~mm}$.

Four series of parallel (the latest solar panels): 320 watts per square meter.

Roof area $\mathrm{S}$ total $=$ length $*$ width $=4.945 * 1.845=9.12$ square meters remove the exhaust fan, assuming the available area is $\mathrm{S}=8$ square meters, then $\mathrm{P}$ too always $=\mathrm{S} * \mathrm{p}=8 * 320=2560$ watts (assuming Sunny, appropriate angle of exposure)

\subsection{Hand-cranked generator}

Now the smartphone is well received by everyone, but it has a drawback short standby time. Therefore, in order to prevent passengers in the process of car phone no electricity, we have in each side of the tables and chairs are added a hand-cranked generator, in its side there is a mobile phone charging line interface, so that people in the car to the phone Charge, the idea to solve the problem of no electricity phone, taking into account the "people-oriented" concept.

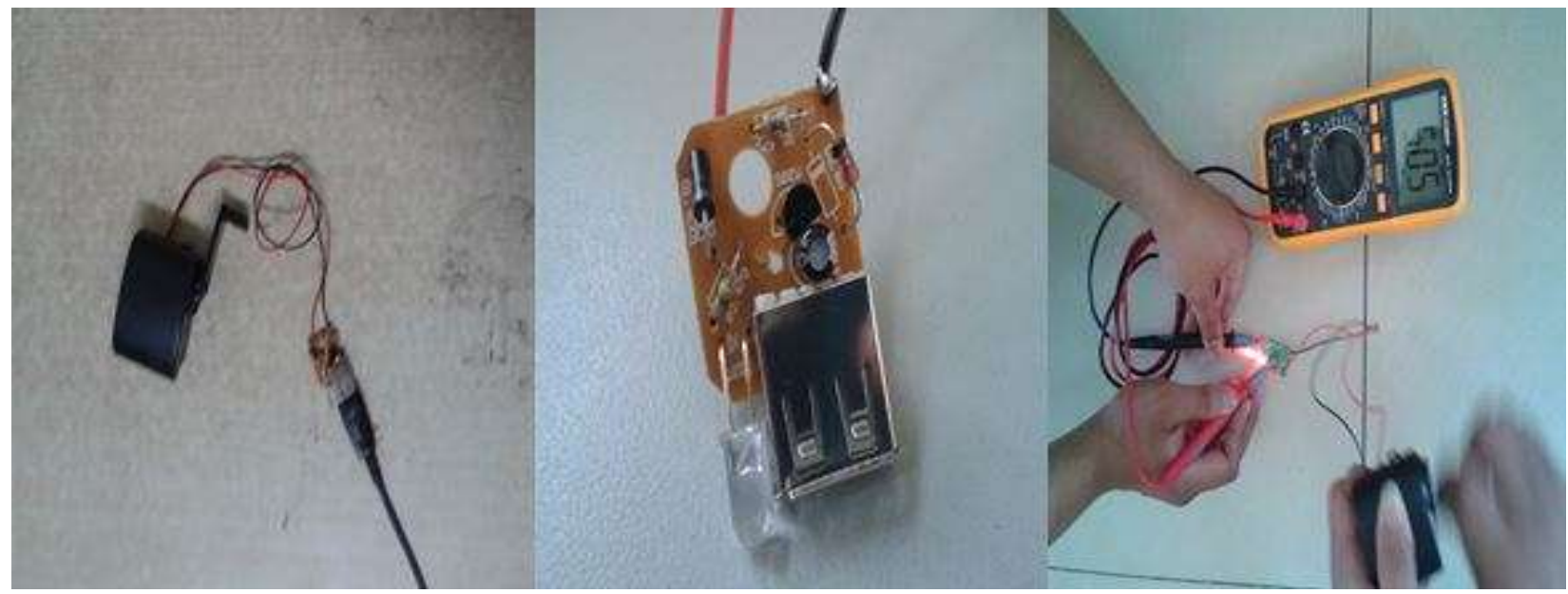

Figure 4: Hand-cranked generator model

In addition, the market now smart phone charging voltage is generally $5 \mathrm{~V}$, we designed the hand-cranked generator in the regulator can stabilize the original voltage at about $5 \mathrm{~V}$ (as above), for the passenger's mobile phone to provide a stable charge Environment, to ensure that the passengers will not burn off the phone or some unnecessary accidents.

\subsection{Resonant electric vehicle car wireless charging system}

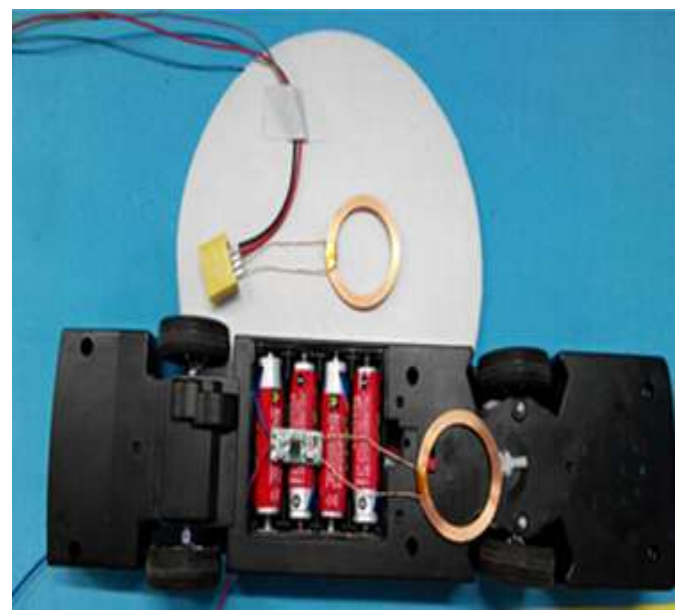

Figure 5: Electric vehicle wireless charging device

\section{The principle and application of super capacitor}

Supercomputer, also is known as large capacity capacitors, gold capacitors, gold capacitors, energy storage capacitors, Faraday capacitors, electrochemical capacitors or electric double layer capacitors, that which is to rely on polarized electrolyte to store electrical energy of the different electrochemical devices. The super capacitor is based on the theory of electrochemical double layer, which belongs to the electric double layer capacitor, which is the largest capacity in the electric double layer capacitor which has been made available for mass production in the world. Principle The two types of conductive plates can be utilized to hold the insulating layer between the plates. The voltage is kept in the plates. When the voltage is implemented between the plates, the charge is kept in the plates. The stored energy can express support for the We, where the diaphragm is the insulating medium; $\Delta \mathrm{V}$ is the volume of the insulating medium; $\mathrm{E}$ is the electric field strength of the insulating medium. Energy consumption per unit volume, ie, energy storage density, can be expressed as:
Electromagnetic resonant coupling radio power transmission technology is the use of magnetic resonance coupling wireless power transmission, power transmission field is a cutting-edge issue, is a new technology, it can be efficient and flexible to achieve wireless transmission of electricity. Electromagnetic resonant radio power transmission technology as a modern energy transmission, charging convenient and quick, to stop the station can quickly charge the request.

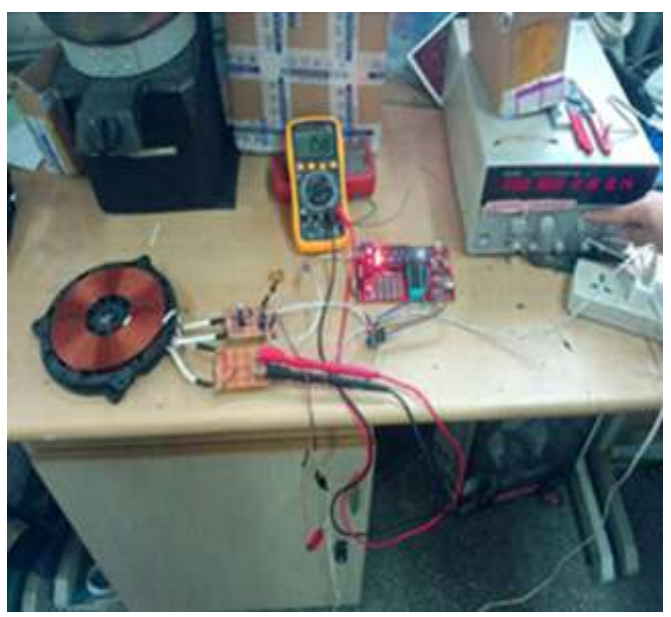

Figure 15 : Resonant wireless energy transfer device

$$
\omega e=\frac{1}{2} \varepsilon E^{2}
$$

It can be seen that increasing the dielectric constant and the electric field strength increases the energy storage density of the capacitor.

Supercomputer usually contain two electrodes, electrolyte, current collector, separator four components, which the use of activated carbon porous electrode and electrolyte composition of the double layer structure to obtain large capacity. When the applied voltage is applied to the two plates of the super capacitor, as with the ordinary capacitor, the positive electrode of the plate stores a positive charge, and the negative plate stores a negative charge. Under the electric field generated by the charge on the two plates of the super capacitor, The opposite charge is formed at the interface between the electrolyte and the electrode to balance the internal electric field in the electrolyte. This positive charge and negative charge are arranged on the contact surface between two different phases with a 
very short gap between positive and negative charges in the opposite position, this charge distribution layer is called an electric double layer, so the capacitance is very large. As the super capacitor discharges, the charge on the positive and negative plates is discharged by the external circuit, and the charge on the interface of the electrolyte is reduced accordingly. It can be seen: the super capacitor charging and discharging process are always a physical process. There is no chemical reaction. Therefore, unlike the battery using the chemical reaction, the performance is stable.

According to the basic framework, we have developed a small renewable energy grid-connected power generation system as showed. The power generation system in the system may be a solar or wind power generation system in which the respective power generation devices are connected together by respective converters to form a DC bus. At the same time, the DC bus through the power electronic devices and inertia flywheel and super capacitor connected to the energy storage device through the corresponding supplement or absorption to adjust the output current tends to a stable level, that is, within a certain range of fluctuations. Then the DC bus through the grid inverter device to get AC, through the switch can be attached to the public grid to achieve power generation, can also be connected to the AC load. This system principle can be implemented to microgrids.

The flywheel energy storage device is mainly used in the regulation of renewable energy power generation system, real-time detection of changes in the momentum of the power grid, real-time control flywheel energy storage device compensation momentum, in order to achieve renewable energy power generation system and flywheel energy storage system energy state of a reasonable description, Real-time computing and energy exchange control, thus achieving the premise of grid. At present, recent research on the generalized momentum compensation control of renewable energy power generation system based on flywheel energy storage technology solves this problem.

\subsection{The generalized momentum model of flywheel energy storage} system is

$$
P_{s}=\frac{\partial T_{s}}{\partial \omega}=J \omega
$$

Control the flywheel energy storage system in a certain period of time with the renewable energy power generation grid energy exchange, energy storage system from one operating state changes to another operating state, with the interaction of the power grid because of the energy or lost energy, Its operating state will change. This process is characterized as: the flywheel energy storage system collides with the grid, and the total momentum remains constant during the collision. Real-time measurement of flywheel energy storage system state parameter $\omega$ size, you can quickly calculate the flywheel energy storage system, instantaneous momentum and a certain amount of time the amount of change in momentum.

3.2 The generalized momentum model of renewable energy power generation system is

$$
P_{r g}=\frac{\partial T_{r g}}{\partial U_{r g}}=C_{r g} U_{r g}
$$

The use of Hall voltage sensors and program-controlled microprocessors can be real-time measurement and rapid calculation of renewable energy power generation system center point voltage Urg, which can quickly calculate the instantaneous momentum of renewable energy power generation system and a certain period of time changes in momentum the amount.

\subsection{Mathematical model of flywheel energy storage}

Assuming that the three-phase potential of the motor is Ea, Eb, Ec, the three-phase winding current is ia, ib, ic, the terminal voltage is $\mathrm{Ua}, \mathrm{Ub}, \mathrm{Uc}$, and the three-phase stator winding adopts star connection. The threephase winding resistance $R a=R b=R c=R$, the three-phase winding selfsensation $\mathrm{La}=\mathrm{Lb}=\mathrm{Lc}=\mathrm{L}$, the three-phase winding resistance is the same as the parameters, the three-phase winding interaction $\mathrm{Lab}=\mathrm{Lba}=\mathrm{Lac}=$ $\mathrm{Lc}=\mathrm{Lbc}=\mathrm{Lcb}=\mathrm{M}, \mathrm{D}$ is the differential operator, and $\mathrm{Un}$ is the common connection point voltage, then the voltage equation can be expressed as follows when running in generator mode:

$$
\left[\begin{array}{l}
U_{a} \\
U_{b} \\
U_{c}
\end{array}\right]=\left[\begin{array}{l}
E_{a} \\
E_{b} \\
E_{c}
\end{array}\right]-\left[\begin{array}{ccc}
R & 0 & 0 \\
0 & R & 0 \\
0 & 0 & R
\end{array}\right]\left[\begin{array}{l}
i_{a} \\
i_{b} \\
i_{c}
\end{array}\right]-\left[\begin{array}{ccc}
L & M & M \\
M & L & M \\
L & M & L
\end{array}\right] D\left[\begin{array}{l}
i_{a} \\
i_{b} \\
i_{c}
\end{array}\right]-U_{n}\left[\begin{array}{l}
1 \\
1 \\
1
\end{array}\right]
$$

When running in motor mode, the voltage equation can be expressed as:

$$
\left[\begin{array}{l}
U_{a} \\
U_{b} \\
U_{c}
\end{array}\right]=\left[\begin{array}{l}
E_{a} \\
E_{b} \\
E_{c}
\end{array}\right]+\left[\begin{array}{ccc}
R & 0 & 0 \\
0 & R & 0 \\
0 & 0 & R
\end{array}\right]\left[\begin{array}{l}
i_{a} \\
i_{b} \\
i_{c}
\end{array}\right]+\left[\begin{array}{ccc}
L & M & M \\
M & L & M \\
L & M & L
\end{array}\right] D\left[\begin{array}{l}
i_{a} \\
i_{b} \\
i_{c}
\end{array}\right]+U_{n}\left[\begin{array}{l}
1 \\
1 \\
1
\end{array}\right]
$$

According to equation (4), the equivalent circuit of the flywheel energy storage device can be established. In the case of a phase, when the motor rotates at an angular velocity $\omega$, the single-phase electromagnetic torque Ta can be expressed as

$$
T_{a}=E_{a} i_{a} / \omega
$$

The total electromagnetic torque $\mathrm{Te}$ is the sum of the three-phase torque

$$
\mathrm{Te}=\left(\mathrm{E}_{\mathrm{a}} \mathrm{i}_{\mathrm{a}}+\mathrm{E}_{\mathrm{b}} \mathrm{i}_{\mathrm{b}}+\mathrm{E}_{\mathrm{c}} \mathrm{i}_{\mathrm{c}}\right) / \omega
$$

When the flywheel energy storage device is operated in the discharge mode, the total torque of the flywheel overcomes the load torque TL, the damping torque $\mathrm{B} \omega$ and the generator total electromagnetic torque $\mathrm{Te}$, the relationship can be expressed as follows:

$$
-\mathrm{J} \frac{\mathrm{d} \omega}{\mathrm{dt}}=\mathrm{T}_{\mathrm{L}}+\mathrm{B} \omega+\mathrm{T}_{\mathrm{e}}
$$

When the motor is running in the motor mode, its mechanical energy equation can be expressed as

$$
\mathrm{T}_{\mathrm{e}}=\mathrm{T}_{\mathrm{L}}+\mathrm{B}_{\omega}+\mathrm{J} \frac{\mathrm{d} \omega}{\mathrm{dt}}
$$

Where J is the flywheel moment of inertia. For cylindrical flywheels, the moment of inertia is:

$$
\mathrm{J}=m r^{2}=\rho \pi r^{2} 1 r^{2}
$$

Where: $\rho$ for the flywheel material density; $r$ for the flywheel radius of rotation; l for the flywheel thickness.

\subsection{Generalized momentum compensation control method}

The generalized momentum conservation theory provides a reliable basis for the energy exchange control between the flywheel energy storage device and the renewable energy power generation system. When the external environmental factors change or load changes, the renewable energy power generation system can be considered with the outside of the energy collision, the original balance of the system has changed, the momentum has changed, the voltage Urg has also undergone a corresponding change, thus affecting the supply voltage quality. Similarly, when the grid due to a short circuit, open circuit and other reasons caused by sudden changes in the grid structure, equivalent inertia parameter $\mathrm{Crg}$ changes, the system to maintain its generalized momentum conservation, Urg will inevitably change accordingly, may exceed the allowable supply voltage range.Each time the central point voltage does not meet the requirements, first a flywheel energy storage device and the power grid between the small momentum collision, measuring the small momentum collision caused by the flywheel speed change $\Delta \omega$ and power grid.The central point of the voltage change $\Delta U$ Urg, after a series of calculations, and finally get the required torque at this time.

$$
T=J \beta=\frac{m r^{2}}{2} \frac{\Delta \omega}{\Delta t}=\frac{\rho \pi r^{2} l r^{2}}{2} \frac{\Delta n \cdot 2 \pi}{\Delta t}
$$


According to the torque obtained by Eq. (9), the change value of the voltage at the output end of the flywheel motor can be obtained by reversing (4) (8) to compensate the bus voltage of $\Delta \mathrm{Urg}$, and then the controllable variable of the control system can be obtained.

\subsection{Flywheel energy storage device control flow chart}

The method of energy colliding with the renewable energy power generation system changes the momentum of the power grid by collision, so that the voltage of the grid center point can the method of energy colliding with the renewable energy power generation system changes the momentum of the power grid by collision, so that the voltage of the grid center point can be restored to the level of power quality requirements. The control flow is shown in Fig. If the grid voltage is reduced, the control system quickly calculates the amount of power loss in the grid and calculates the same magnitude of the flywheel output to reduce the required speed and time and adjust the flywheel energy storage device. If the grid voltage increases, the control system quickly calculate the amount of power fluctuations in the grid, while calculating the flywheel to obtain the same size of the momentum needs to increase the speed and time and the flywheel energy storage device to accelerate energy storage adjustment.

Experiments show that, regardless of whether the solar photovoltaic power generation system is subject to external interference or internal network changes, flywheel energy storage devices can be real-time to the photovoltaic power generation system to provide appropriate compensation momentum, the photovoltaic power generation system voltage quickly and accurately adjust to ensure that the central point voltage stable.

Inverter is a photovoltaic device, fan, fuel cell, energy storage components through the power electronic switch and micro-network system connected to the crucial equipment. The converter may include both a rectifier and an inverter, or only an inverter. The converter has the characteristics of fast speed, low inertia and weak overcurrent capacity. At the same time, suitable for micro-network inverter in addition to the need for conventional inverter function and can run in parallel but being also dependent on the special needs of micro-network system with some control functions. The structure and operation of the inverter are the major technologies in the microgrid.

Widely used in pure electric vehicle energy supply system - super capacitor battery compared with other batteries, charging time is short, charge and discharge cycle long life, high quality than high power, high reliability, in the vehicle design and development process, its Single cell arrangement of the connection not only to meet the performance requirements of the vehicle, but also in the energy recovery process to ensure that the battery safe, as much as possible to store more energy. In the energy recovery process, the pure electric vehicle super capacitor energy storage system's principal role is to quickly and effectively absorb more braking energy.Be returned to the level of power quality requirements. The control flow is shown in Fig. If the grid voltage is reduced, the control system quickly calculates the amount of power loss in the grid, and calculates the same magnitude of the flywheel output to reduce the required speed and time and adjust the flywheel energy storage device. If the grid voltage increases, the control system quickly calculate the amount of power fluctuations in the grid, while calculating the flywheel to obtain the same size of the momentum needs to increase the speed and time and the flywheel energy storage device to accelerate energy storage adjustment.

Experiments show that, regardless of whether the solar photovoltaic power generation system is subject to external interference or internal network changes, flywheel energy storage devices can be real-time to the photovoltaic power generation system to provide appropriate compensation momentum, the photovoltaic power generation system voltage quickly and accurately adjust to ensure that the central point voltage stable.

Inverter is a photovoltaic device, fan, fuel cell, energy storage components through the power electronic switch and micro-network system connected to the crucial equipment. The converter may include both a rectifier and an inverter, or only an inverter. The converter has the characteristics of brisk speed, low inertia and weak overcurrent capacity. At the same time, suitable for micro-network inverter in addition to the need for conventional inverter function and can run in parallel but being also dependent on the special needs of micro-network system with some control functions. The structure and operation of the inverter are the main technologies in the microgrid.

Widely used in pure electric vehicle energy supply system - super capacitor battery compared with other batteries, charging time is short, charge and discharge cycle long life, high quality than high power, high reliability, in the vehicle design and development process, its Single cell arrangement of the connection not only to meet the performance requirements of the vehicle, but also in the energy recovery process to ensure that the battery safe, as much as possible to store more energy. In the energy recovery process, the pure electric vehicle super capacitor energy storage system's principal role is tantamount to quickly and effectively absorb more braking energy.

In order to meet the requirements, the super capacitor energy storage system must have sufficient capacity to store the maximum braking energy, and in order to meet the safety requirements of the super capacitor energy storage process, but also consider the regenerative braking process peak power. Electric taxi model using rear wheel drive, model taxi motor for permanent magnet DC brush motor, model electric bus power supply for the $41.2 \mathrm{~V}$ lithium battery, super capacitor consists of two 2.5V600F super capacitor series, each Super capacitor at both ends and a voltage regulator circuit to ensure that the super capacitor can be stable and efficient absorption of energy, super capacitor and power supply between the lithium battery through the chopper circuit and the use of rated current charging method of lithium battery charging device phase connection.

\section{INNOVATION ADVANTAGE}

The use of super capacitor and flywheel energy storage of these two advanced energy storage methods as a new energy power generation system in the energy storage means, not only real-time detection, compared with other means of storage, the more accurate to add momentum or absorb momentum to achieve a smooth Transmission. And in the high efficiency, safety, conditions of use, life and so on, there is irreplaceable superiority. In addition, these two energy storage methods clean and pollution-free, and technology and more mature, which is everything a matter of very good development prospects.

Solar thermal power generation vehicle roof and two total generations in power co-generation unit of up to $12 \mathrm{KW}$, fully provides real-time source of energy for the electric taxis. To ensure that the electric taxi operation of the safe and reliable, to ensure the real-time influx of energy, green, energy saving.

\section{CONCLUSION}

The use of super capacitor and flywheel energy storage of these two advanced energy storage methods as a new energy power generation system in the energy storage means, not only real-time detection, compared with other means of storage, the more accurate to add momentum or absorb momentum to achieve a smooth Transmission. And in the high efficiency, safety, conditions of use, life and so on, there is irreplaceable superiority. In addition, these two energy storage methods clean and pollution-free, and technology and more mature, has a very good development prospect.

\section{REFERENCE}

[1] Jiancheng, Z., Lipei, H., Zhiye, C., Su, W. 2003. Study on flywheel energy storage system and its control technology [J]. Proceedings of the Chinese society of electrical engineering, 23 (3), 108-111.

[2] Lijun, Q., Han, Z., Lixin, G. 2002. Key technologies of the development of electric vehicle [J]. Journal of HeFei University of Technology (NATURAL SCIENCE EDITION), 25 (1), 14-18.

[3] Ximing, Fengchun, S. 2001. Electric vehicle overview of energy storage technology [J]. Power technology, 25 (1), 47-52.

[4] Junling, C., Xin, J.X., Dongqi, Z. 2005. Control strategy of new type flywheel UPS motor [J]. Journal of Tsinghua University, 4l (6): 188-193.

[5] Chao, W., Wei, J. 2010. Application of flywheel energy storage system in automobile [J]. Mechanical design and manufacture, (12), 82-84.

[6] Xingjian, D., Han, Y., Yiliang, L. 2009. Experimental study on permissible discharge efficiency of flywheel energy storage system [J]. Transactions of China Electrotechnical Society, 24 (3), 20-24[7]. (in Chinese)

[7] Shuangqing, T., Daoxun, L. 2003. The core technology and development prospect of electric vehicle [J]. Mechanical Science and Technology, (3): 189-192. 
[8] Shuangqing, T. 2007. Flywheel energy storage technology and application [M]. Wuhan: Huazhong University of Science and Technology press.

[9] Zhiyong, D., Xingjian, D. 2006. Study on the no-load loss of flywheel energy storage system with spiral groove cone bearing [J]. Mechanical Science and Technology, 25 (12), 1434-1437. (in Chinese)
[10] Chinese, W., Xingjian, D., Zupei, S. 2005. Journal of energy storage flywheel wind damage of the theoretical calculation and Experimental Research on [J]. Mechanical Engineering, 41 (6), 188-193.

[11] Ciwei, G., Liang, Z. 2011. The impact on power system of electric vehicle charging in [J]. Electrical Engineering College of Southeast University. Power system technology, (2). 